\section{El Profesor Doctor Hernán Alessandri Rodríguez (1900-1980)}

\author{
HUMBERTO REYES B.
}

\section{Professor Hernán Alessandri, M.D. (1900-1980)}

Hernán Alessandri M.D. was an astounding clinician and a leading medical educator, born in Santiago in 1900 where he died in 1980. He received his medical degree at the University of Chile in 1923, became Professor of Clinical Medicine in 1932, Full Professor and Chair of Internal Medicine in 1944. At the Hospital del Salvador, in Santiago, he chaired a teaching Department and a Clinical Service that was an example for its academic environment and dedication to patients and students. From 1958 to 1962 he was Dean of the University of Chile Faculty of Medicine, conducting a reform of teaching curricula and organizing medical residency programs for the training of specialists, originally started in his own Service in 1952. The American College of Physicians awarded him the first foreign Honorary Membership. He was a founding Member of the Chilean Academy of Medicine (1964). In 1973 the University of Chile awarded him the Emeritus Professor status. He was considered by his peers, alumni and patients a brilliant clinician and an exceptional medical educator. Since 1980 a Social and Teaching Foundation bears his name and in 2014, with the occasion of the XXXV Chilean Congress of Internal Medicine, the Sociedad Médica de Santiago-Chilean Society of Internal Medicine created an annual lecture to render tribute to distinguished physicians and his name was one of the two selected to inaugurate them.

(Rev Med Chile 2015; 143: 101-108)

Key words: Chile; Education, medical; Famous persons; History of Medicine, 20th Century.

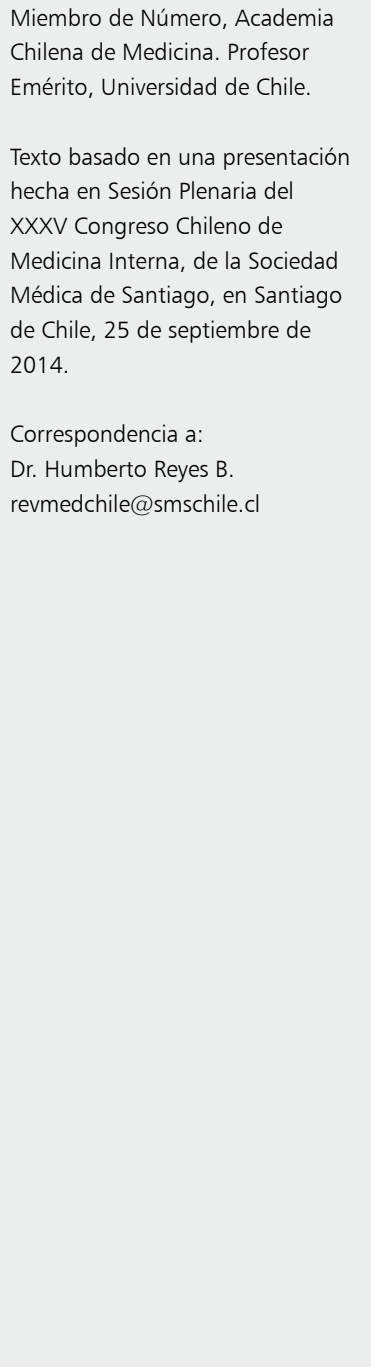

E 1 Profesor Doctor Hernán Alessandri Rodríguez dedicó su vida a la medicina, centrándola en cuatro instituciones: el Hospital del Salvador, la Facultad de Medicina de la Universidad de Chile, la Sociedad Médica de Santiago y la Academia Chilena de Medicina. El texto siguiente, basado en publicaciones previas $^{1-4}$, sintetiza su biografía y agrega algunos comentarios, sesgados por un inevitable conflicto de intereses: llegué al Hospital del Salvador en 1956, como alumno de $4^{\circ}$ año de medicina y desde entonces ese hospital ha sido el hogar de mi carrera clínica asistencial.

\section{Antecedentes}

Entre 1930 y 1960 la medicina alcanzó en Chile un nivel de excelencia ejemplar en el ámbito latinoamericano. Esto ocurría en un país muy alejado de los grandes centros en que se generaba el progreso, cuando las comunicaciones eran lentas y con tecnología que muchos lectores tal vez no conocieron ni por su nombre, como es el caso del telégrafo, con alfabeto Morse. Las llamadas telefónicas internacionales necesitaban conectarse a través de cables submarinos que iban de uno a otro continente. Un viaje desde Chile a los EEUU o a Europa, en 


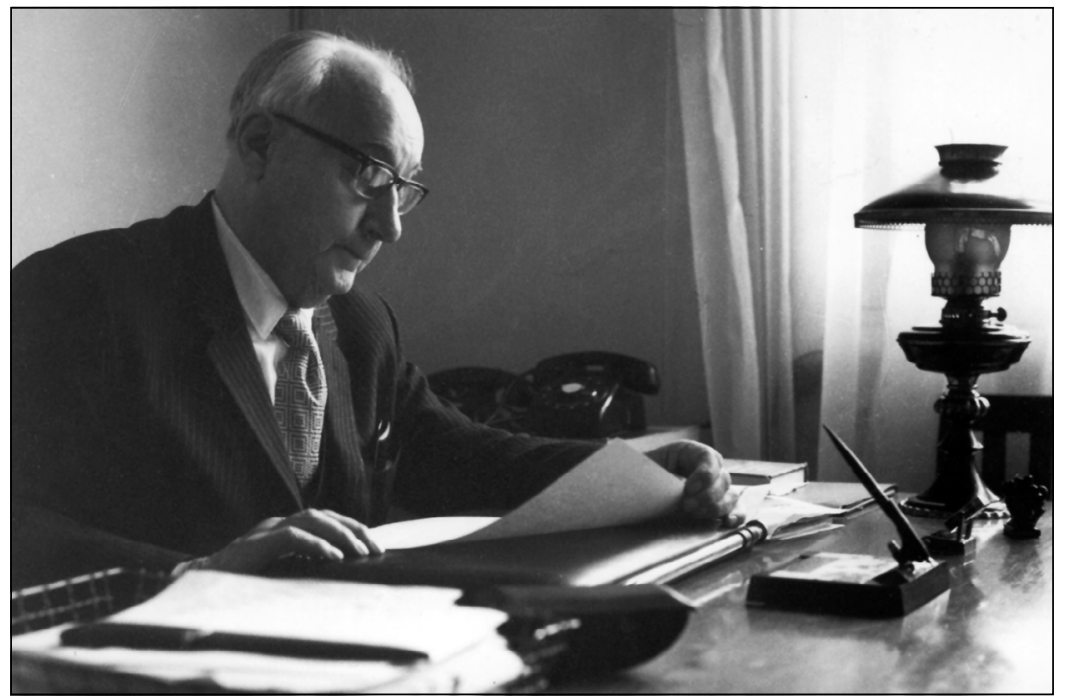

Fotografía 1. El doctor Hernán Alessandri Rodríguez, en 1960. avión con motores con hélices, demoraba dos días. Las revistas médicas llegaban a Santiago por vía marítima, dos a tres meses después de haber sido publicadas en Europa o Norteamérica. Los manuscritos y las cartas se escribían en "máquinas de escribir" marca Underwood, Remington u otras. Sin embargo, en esa época la medicina en Chile se aproximó enormemente a lo que estaba disponible en los países desarrollados. Para que esto ocurriera fue crucial que existieran individuos inteligentes, visionarios, tenaces y amantes de su profesión.
En este aspecto, una de las personalidades más destacadas fue don Hernán Alessandri Rodríguez.

\section{Niñez y juventud (Tabla 1)}

El doctor Alessandri nació en Santiago, en el seno de una familia que dio origen a personalidades distinguidas en el servicio público y el desarrollo de la nación. Su padre, don Arturo Alessandri Palma y posteriormente uno de sus hermanos, don

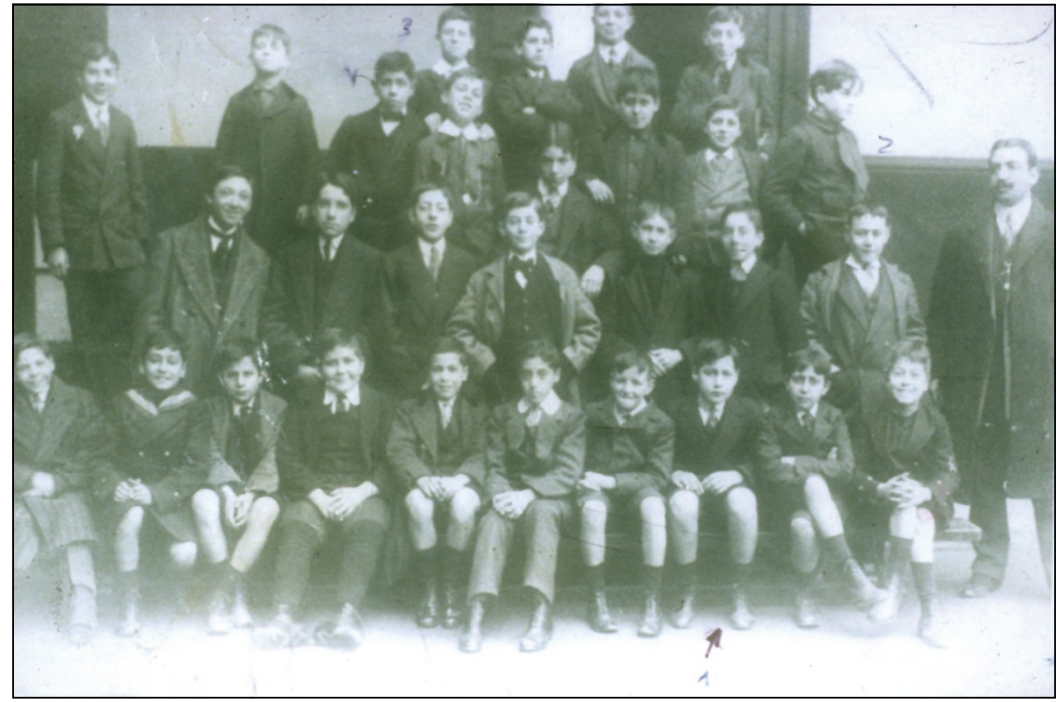

Fotografía 2. En el Instituto Nacional, curso de enseñanza básica ("Primera Preparatoria"). En primera fila, señalado por una flecha, el niño Hernán Alessandri. 

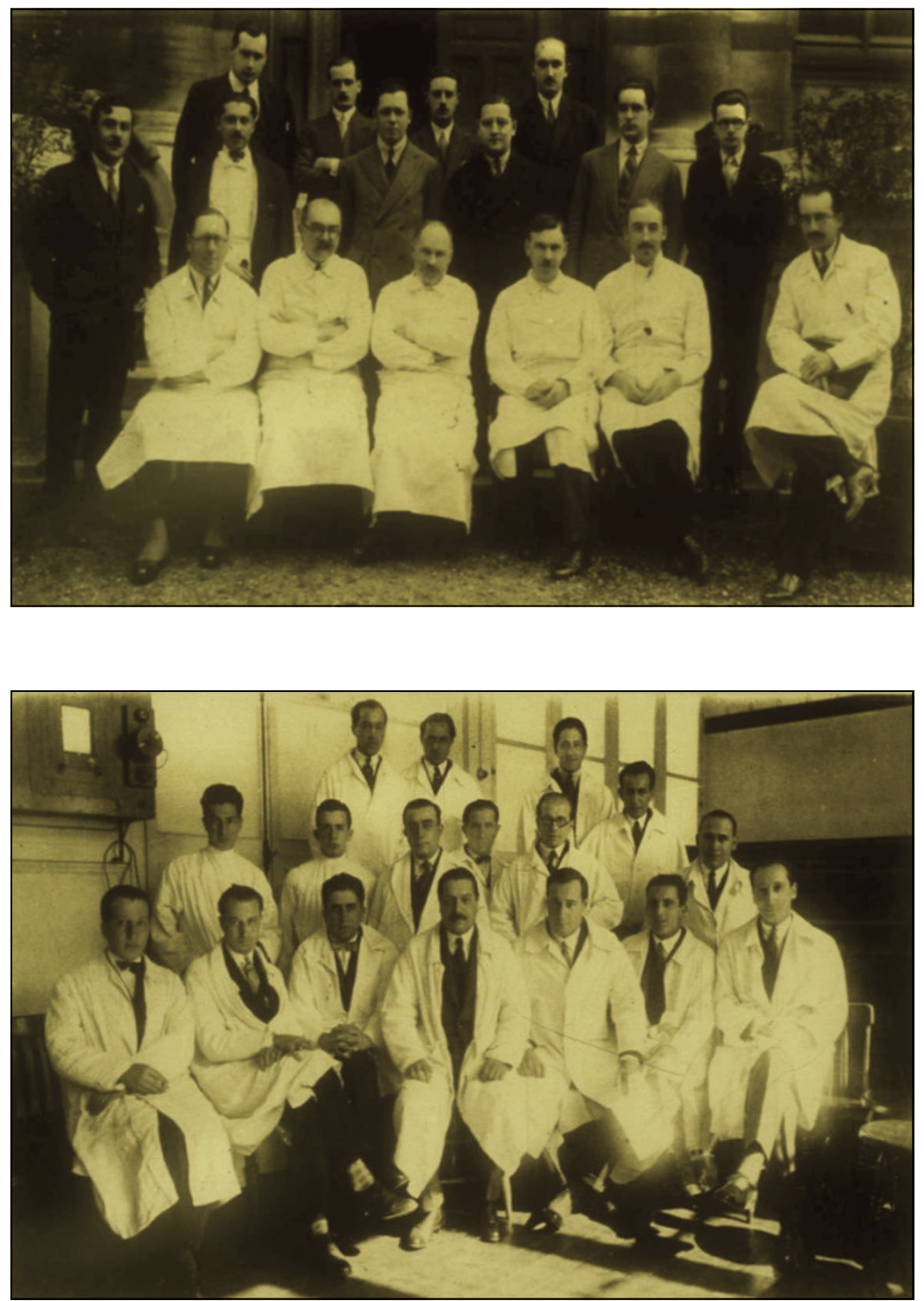

Fotografía 3. En un hospital de París. En primera fila, los profesores. Atrás, a la izquierda, el doctor Alessandri, visitante.
Fotografía 4. En el Hospital San Vicente: médicos de la Clínica del Profesor Ernesto Prado Tagle (al centro) y a su izquierda el doctor Hernán Alessandri.
Jorge Alessandri Rodríguez, fueron Presidentes de la República y marcaron hitos importantes en la historia de Chile.

Estudió en el Instituto Nacional (Fotografía 2) y en la Universidad de Chile, titulándose de médico-cirujano a los 23 años de edad, con el premio al mejor alumno de su promoción. Se incorporó como ayudante en la Clínica Médica del Hospital San Vicente de Paul, que décadas después daría origen al Hospital Clínico de la Universidad de Chile.

\section{Tabla 1. Niñez y juventud}

\begin{tabular}{|ll|}
\hline 1900 & Nace en Santiago de Chile \\
1908 & Instituto Nacional, Primera Preparatoria \\
1917 & Escuela de Medicina de la Universidad de Chile \\
1923 & Médico Cirujano: Premio Clin al mejor egresado \\
$1927-1929$ & Deportado con su familia a París \\
1931 & $\begin{array}{l}\text { Renuncia al Hospital San Vicente, se incorpora } \\
\text { al Hospital del Salvador }\end{array}$ \\
1931 & Profesor Extraordinario de Patología Médica \\
1937 & Profesor Titular de Semiología \\
\hline
\end{tabular}




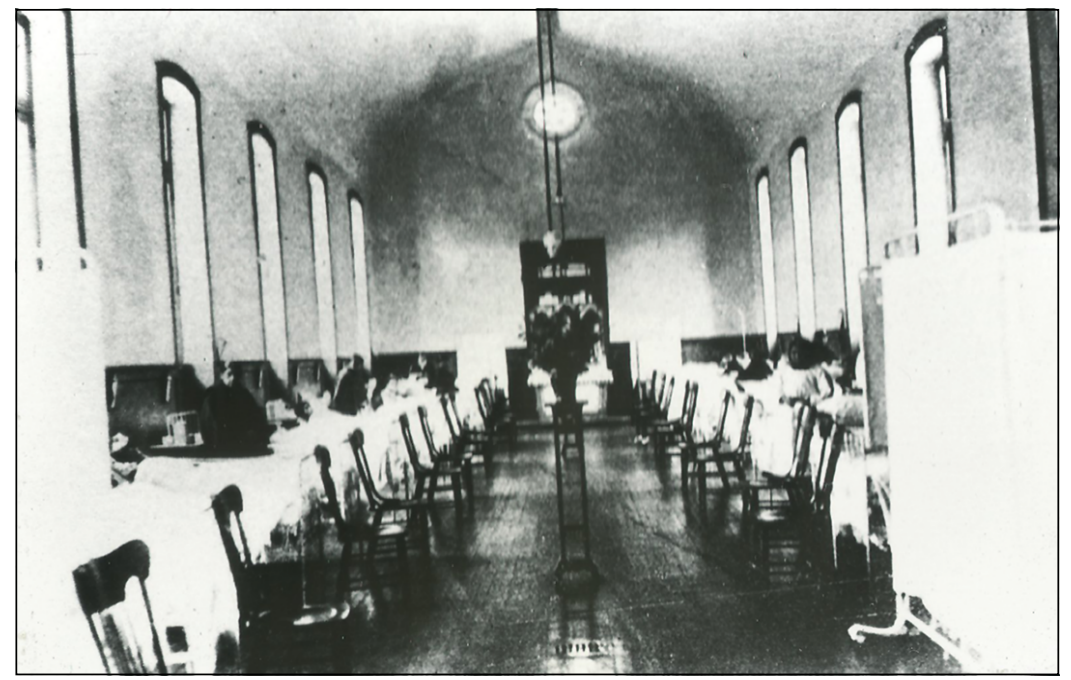

Fotografía 5. Hospital del Salvador, circa 1930: una sala de hospitalización para mujeres. Nótese el brillo del piso de madera, impecables cubrecamas blancas y elegantes sillas.
Siendo alumno de medicina consiguió incorporarse a un turno en la Asistencia Pública, para aprender a enfrentar urgencias médicas, actividad que mantuvo en los primeros años de profesión. Asimismo, asistía a las reuniones de la Sociedad Médica de Santiago, presenciando las discusiones de casos y experiencias clínicas entre destacados clínicos.

En 1927, la situación política nacional lo forzó a exiliarse en Europa, junto con sus padres y hermanos. En Francia y Alemania asistió a clínicas médicas prestigiosas, donde vislumbró el nacimiento de las subespecialidades de la Medicina Interna (Fotografía 3).

Al regresar del exilio se reubicó en el Hospital San Vicente de Paul, que concentraba a la mayoría de los docentes universitarios. Recuperó su cargo en la Clínica Médica que dirigía el Profesor Doctor Ernesto Prado Tagle (Fotografía 4).

Sin embargo, la personalidad fuerte e independiente del doctor Alessandri y su enorme capacidad de estudio generó allí un ambiente incómodo para su vida académica. Sus colegas resentían la atracción que ejercía sobre los médicos jóvenes y los alumnos de medicina, que preferían acompañarlo en sus visitas a pacientes hospitalizados. Por eso, en 1930 decidió retirarse del Hospital San Vicente y fue acogido en el Hospital del Salvador por los doctores Rodrigo Donoso e Israel Bórquez Silva. Hacia allá lo siguieron alumnos y médicos jóvenes.

En esa época el Hospital del Salvador era una institución asistencial (Fotografía 5). Pocos estudiantes lo elegían para sus prácticas clínicas, porque no encontraban docentes de gran nivel. La Facultad de Medicina nombró Profesor Titular de Semiología al doctor Alessandri, a los 37 años de edad, y creó la cátedra respectiva en este hospital.

El diario El Mercurio de Santiago, en su edición del martes $1^{\circ}$ de junio de 1937, publicó un aviso llamando a concurso para proveer el cargo de médico jefe de Medicina Interna en el Hospital del Salvador, "con una renta mensual de \$ 525 más el 25\% de gratificación" (sumando un total de \$ 656,25 , equivalentes actualmente a $\$ 83.668$ ). El ganador fue el doctor Alessandri, quien ejerció dicha jefatura durante 21 años, hasta su elección como Decano de la Facultad de Medicina.

La llegada del doctor Alessandri y otros distinguidos médicos a distintas especialidades empezó a cambiar la imagen del Hospital del Salvador hasta transformarlo en un centro asistencial y docente de gran atractivo.

El Servicio de Medicina pasó a ser un modelo del progreso en la asistencia médica y la enseñanza de la medicina. Otros Servicios del hospital también progresaron, estimulados por ese ejemplo, y los nombres de sus conductores fueron muy respetados: en Cirugía, Manuel Martínez y Félix de Amesti; en Obstetricia, Víctor Manuel Avilés y Luis Tisné; en Oftalmología, Carlos Charlín; en Anatomía Patológica, Ismael Mena, Roberto Barahona y Sergio Donoso; en Urología, Carlos Lobo Onell. Alfonso Asenjo fue fundador del Instituto 


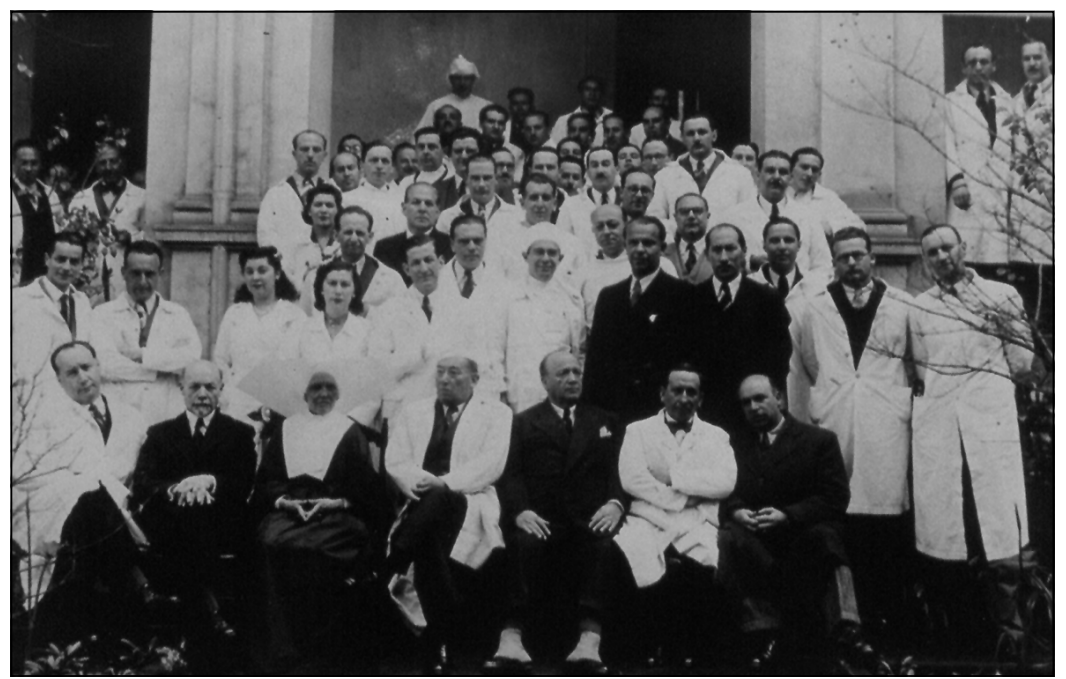

Fotografía 6. Hospital del Salvador, 1945: médicos de distintas especialidades. En primera fila, a izquierda, el doctor Alessandri. de Neurocirugía y Héctor Orrego Puelma lo fue del Hospital del Tórax (Fotografía 6). Estos líderes y sus colaboradores convirtieron al Hospital del Salvador en un lugar apreciado por los estudiantes que ambicionaban recibir una formación clínica de la mejor calidad.

\section{Madurez (Tabla 2)}

En la Sociedad Médica de Santiago fue su Presidente en dos períodos (1940-1941 y 1951-1953), lo que no ha tenido parangón. Décadas después la Sociedad lo nombró Miembro Honorario. A los 44 años, la Facultad de Medicina de la Universidad de Chile lo nombró Profesor Titular de la Cátedra "E" de Medicina.

Con clara percepción advirtió la influencia creciente de la medicina anglosajona sobre los aspectos científicos, sociales y educacionales de la profesión. Entonces estimuló a discípulos destacados para perfeccionarse en Norteamérica y Europa y contribuir a formar en Chile las subespecialidades médicas.

En su actividad clínica recurría a la nueva tecnología y a los avances de las subespecialidades para sustentar un diagnóstico y elegir la terapéutica adecuada, pero sostenía firmemente que al atender pacientes la Medicina Interna es una especialidad indivisible y exigía que sus colaboradores mantuvieran su rol como internistas, en responsabilidades asistenciales y docentes. Cuando ocurría que frente a un paciente hospitalizado los especialistas habían agotado los exámenes sin llegar a un diagnóstico coherente, solía decirnos: "Hijo, hay que empezar todo de nuevo y tenemos que empezar por lo más importante". Entonces, tomaba una silla, se sentaba junto al enfermo y le pedía que relatara la anamnesis, una vez más.

En el curso de sus años de madurez, el doctor Alessandri adquirió una imagen imponente, en que el intelecto concordaba con su prestancia física y su fuerte personalidad. Sin embargo, era afable con sus buenos alumnos, y muy respetuoso y cariñoso con los pacientes. Despertaba fácilmente

\section{Tabla 2. Madurez}

\begin{tabular}{|c|c|}
\hline 1940-1941 & $\begin{array}{l}\text { Presidente de la Sociedad Médica de San- } \\
\text { tiago }\end{array}$ \\
\hline 1951-1953 & $\begin{array}{l}\text { Presidente por segundo período de la Socie- } \\
\text { dad Médica de Santiago }\end{array}$ \\
\hline 1957 & Profesor Titular de Medicina \\
\hline 1958-1963 & $\begin{array}{l}\text { Decano de la Facultad de Medicina de la } \\
\text { Universidad de Chile }\end{array}$ \\
\hline 1960 & $\begin{array}{l}\text { "Declaración de Principios sobre Objetivos } \\
\text { de la Educación Médica" }\end{array}$ \\
\hline 1957-1975 & $\begin{array}{l}\text { Consejo Asesor de Investigación Médica, } \\
\text { Oficina Panamericana de la Salud }\end{array}$ \\
\hline 1964 & $\begin{array}{l}\text { Miembro Fundador y de Número de la Aca- } \\
\text { demia Chilena de Medicina }\end{array}$ \\
\hline 1967 & $\begin{array}{l}\text { Miembro Académico de la Facultad de Me- } \\
\text { dicina de la Universidad de Chile }\end{array}$ \\
\hline
\end{tabular}



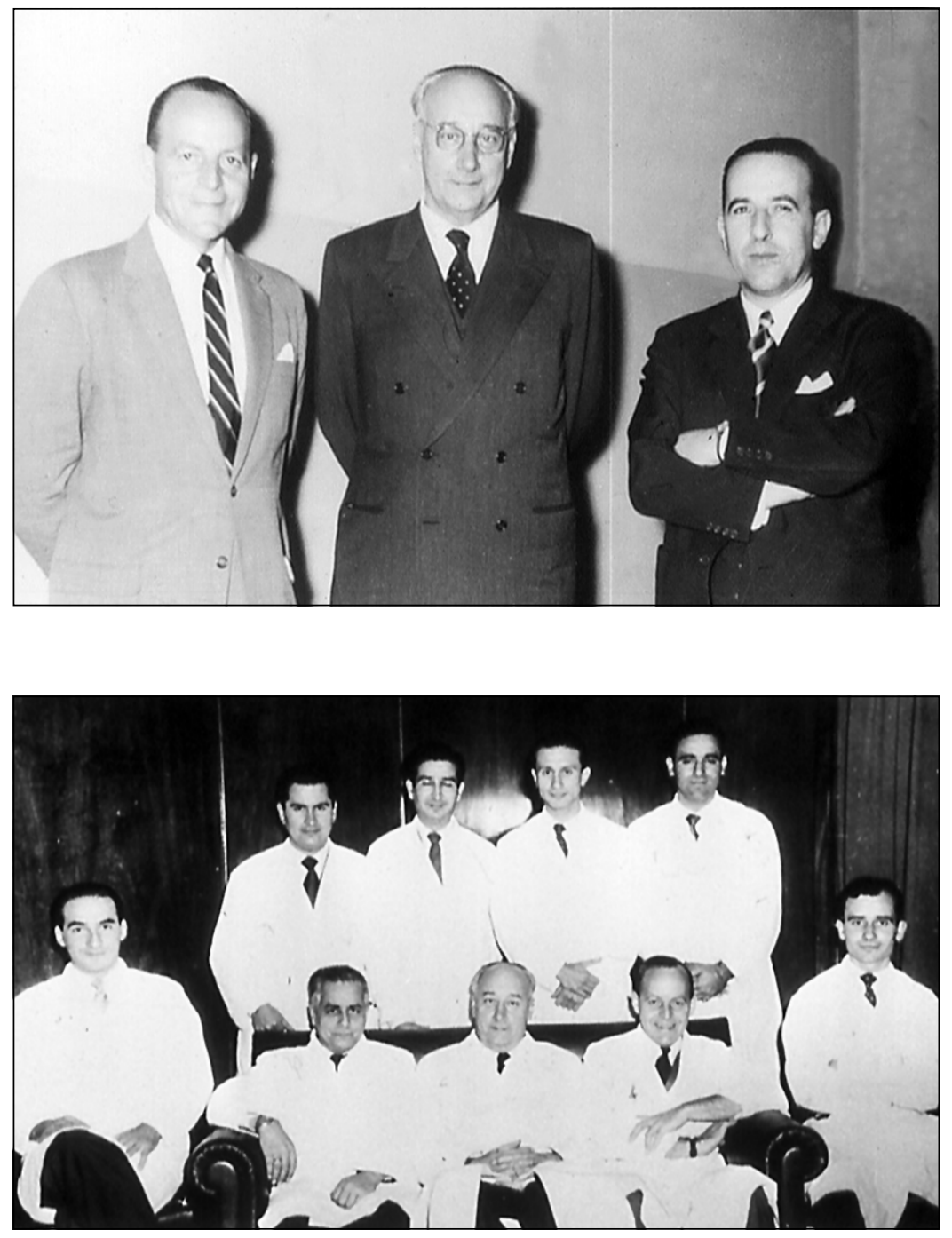

Fotografía 7. El doctor Hernán Alessandri, en 1958, con los doctores Héctor Ducci C. (a su derecha) y Renato Gazmuri O. (a su izquierda).

Fotografía 8. El doctor Hernán Alessandri con los doctores Pedro García Palazuelos (a su derecha) y Héctor Ducci Claro (a su izquierda), rodeados por la segunda promoción de becarios de Medicina Interna. De izquierda a derecha, los doctores Hernán Noguera, Juan Durán, Gustavo D'Acuña, Eduardo Mai, Azaría Dueñas y Alejandro Goic.

esa confianza que es indispensable para la buena relación del médico con su paciente, del profesor con su discípulo, del jefe con su subordinado.

Otros médicos prominentes, contemporáneos suyos, propiciaron ideales análogos e implantaron cambios similares en distintos hospitales docentes. Pero, el mérito de don Hernán fue la fortaleza con que transmitió esta filosofía, la constancia con que practicaba y difundía su concepción de la medicina. Nadie como él fue capaz de presentarla con tal vehemencia y profunda convicción, en sus intervenciones en la Facultad de Medicina y en la Sociedad Médica de Santiago.

A los 58 años de edad fue elegido Decano de la
Facultad de Medicina de la Universidad de Chile y necesitó traspasar la jefatura del Servicio y de la Cátedra a sus discípulos. El doctor Héctor Ducci Claro, de quien se esperaba la primera opción, fue fulminado por un infarto cardíaco a los 43 años de edad. Finalmente, lo sucedió el doctor Renato Gazmuri, un brillante nefrólogo (Fotografía 7).

Durante su decanato consolidó la institución del Internado y de las Becas-residencia para la formación de especialistas. Su Servicio acogió a los primeros becarios de Medicina Interna, seleccionados por concurso público y financiados por el Ministerio de Salud, inaugurando un sistema que se extendió rápidamente a todas las espe- 


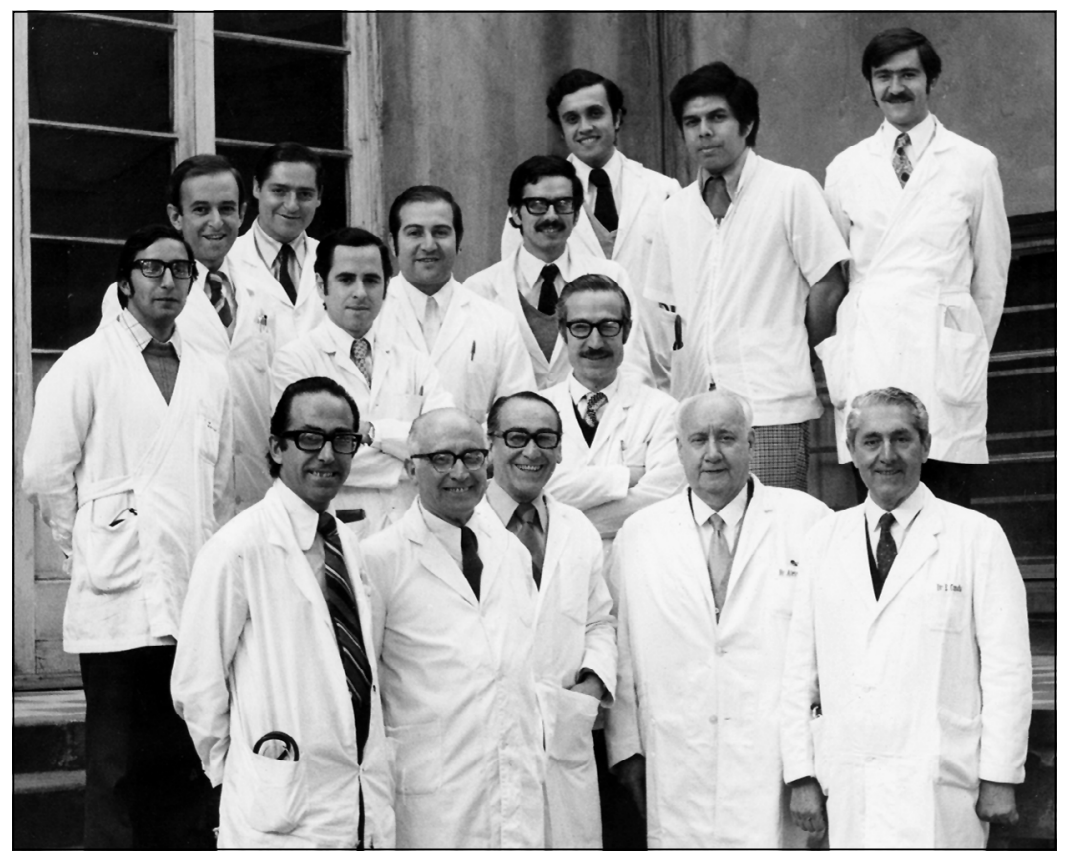

Fotografía 9. Docentes y becarios en 1978. Abajo, de izquierda a derecha: doctores Edgardo Escobar C. (Director del Departamento Docente), Ramón Florenzano G. (Jefe del Servicio de Medicina), Gastón Chamorro Z., Humberto Reyes B. (Subjefe del Servicio de Medicina), Hernán Alessandri R. y Eliseo Concha P. Atrás, los becarios doctores Fernando Lanas, Juan Del Mastro, Antonio Raffo, Juan Enrique Blümel, Jorge Prieto, Jaime Goich, Fernando Florenzano U., Luis Hernández (médico de Honduras) y Ricardo Rademacher. cialidades de la Medicina (Fotografía 8). Junto a su gran colaborador y sucesor en el decanato -el Profesor Amador Neghme- contribuyó a que la Facultad definiera los objetivos para la formación del médico en nuestro país, sintetizados en un documento que ha conservado su importancia hasta la actualidad.

En 1964 fue uno de los Fundadores de la Academia de Medicina del Instituto de Chile.

Desde 1957 hasta 1975 fue miembro del Consejo Asesor de Investigación de la Organización Panamericana de la Salud, asistiendo a reuniones semestrales o anuales en su sede en Washington, EE UU de NA.

\section{Vejez y Fin (Tabla 3)}

Muchas sociedades médico-científicas en el país y en el extranjero se preciaron de tenerlo entre sus miembros. El American College of Physicians, entidad que representa a la Medicina Interna en los Estados Unidos, lo nombró Miembro Honorario y fue el primer médico extranjero que recibió esa distinción.

A los 73 años la Universidad de Chile lo nombró Profesor Emérito.

En 1975 se inició una Conferencia Anual en su homenaje, que ha sido dictada ininterrumpidamente por sus más brillantes discípulos y por destacadas personalidades de la medicina y la salud pública.

En el atardecer de su vida siguió viniendo al hospital, para contribuir a la enseñanza de estudiantes y becados (Fotografía 9). Cito un párrafo escrito por el doctor Alejandro Goic4: "En los últimos años de su vida y por aquellos inescrutables designios del destino, la luz de su brillante inteligencia, que había iluminado a la medicina nacional durante décadas, se fue apagando dolorosa y paulatinamente, falleciendo en Santiago en 1980 después de una prolongada enfermedad".

\section{Tabla 3. Vejez y Fin... ¿Final?}

$\begin{array}{cl}1968 & \begin{array}{l}\text { Miembro Honorario del American College of } \\ \text { Physicians }\end{array} \\ 1973 \text { Profesor Emérito de la Universidad de Chile } \\ 1975 \text { "I Conferencia Anual Doctor Hernán Alessandri } \\ \text { Rodríguez" }\end{array}$


Tabla 4. Presidentes de la Sociedad Médica de Santiago, discípulos del Doctor Hernán Alessandri

\begin{tabular}{|ll|}
\hline $1955-1957$ & Héctor Ducci Claro \\
\hline $1961-1963$ & Renato Gazmuri Ojeda \\
\hline $1967-1969$ & Ricardo Katz Ugarte \\
$1975-1977$ & Marta Velasco Rayo \\
$1985-1987$ & Gustavo Pineda Valdivia \\
$1991-1993$ & Emilio Roessler Bonzi \\
\hline
\end{tabular}

Sus restos fueron velados en la capilla del Hospital del Salvador. A la ceremonia de despedida asistió una multitud de personalidades, representantes de universidades y sociedades médicas científicas, amigos, personal médico, de otras profesiones de la salud y funcionarios del hospital. La misa fue concelebrada por tres sacerdotes, participando su propio hijo, el Padre Hernán Alessandri Morandé. Su fallecimiento fue destacado en crónicas y homenajes en la prensa nacional y en las revistas médicas. Cabe recordar algunas frases de una editorial dedicada póstumamente por su amigo, el Profesor Doctor Rodolfo Armas Cruz

"Su gran personalidad, su inteligencia, su buen criterio médico, lo hacían aparecer como un guía de su generación". "Era algo así como el árbitro de las circunstancias y resultaba como un verdadero caudillo de los grupos médicos".

"Todo eso valía mucho, pero para mí su mayor mérito fue el de luchar por levantar y poner al día la Medicina Chilena, crear una emulación que levantó un ambiente de competencia de estudio y de progreso y que tuvo un solo ganador: la Medicina Chilena".

Esas palabras, escritas por otro gran profesor, retratan a un Maestro que le dio dignidad y prestigio a la medicina chilena.

\section{Más allá del término de una vida}

Para prolongar su recuerdo en el tiempo, un grupo de familiares y amigos, en el país y el extranjero, crearon la Fundación Social y Educativa Doctor Hernán Alessandri Rodríguez, que otorga becas para la capacitación de médicos y premia a jóvenes que se han destacado durante su formación de post-título en el Servicio de Medicina que dirigió el Profesor Hernán Alessandri.

La antorcha encendida por el doctor Hernán
Alessandri y sus ilustres contemporáneos se ha mantenido en las instituciones que él tanto amó, a través de sus discípulos y los discípulos de sus discípulos: seis han sido Presidentes de la Sociedad Médica de Santiago-Sociedad Chilena de Medicina Interna (Tabla 4); cinco han sido Editores de la Revista Médica de Chile; dos han sido Decanos de la Facultad de Medicina: el doctor Alejandro Goic y la doctora Cecilia Sepúlveda; dos ex alumnos de su Cátedra fueron elegidos Rectores de la Universidad de Chile: el doctor Jaime Lavados y el actual Rector, doctor Ennio Vivaldi.

Un texto de Semiología Médica que sigue la doctrina docente del doctor Alessandri, está dedicado a su nombre y se encamina a su cuarta edición, liderado desde su inicio por el doctor Alejandro Goic.

La Sociedad Médica de Santiago quiso revivir en su reciente Congreso anual el recuerdo de uno de sus miembros más preclaros. La Revista Médica de Chile acoge este homenaje a un gran médico, para resaltar su obra ante las generaciones presentes y futuras. Como complemento, se publica a continuación el testimonio presentado por un joven médico que no conoció al doctor Alessandri, pero actualmente está becado para formarse como especialista en Medicina Interna, en el Servicio de Medicina que organizó y dirigió el Profesor Hernán Alessandri.

\section{Referencias}

1. Neghme A. Hernán Alessandri Rodríguez. Médico, Maestro, Académico y Universitario. Su vida y su obra. Editorial Universitaria-Santiago de Chile: 1982.

2. Reyes H. Remembranzas de los últimos años de su cátedra. En: Neghme A. Hernán Alessandri Rodríguez. Médico, Maestro, Académico y Universitario. Su vida y su obra. Editorial Universitaria-Santiago de Chile: 1982. (Págs. 149-152).

3. Goic A. El Dr. Alessandri que yo conocí. Rev Med Chile 1982; 110: 593-5.

4. Goic A. Semblanza del Profesor Dr. Hernán Alessandri R. Rev Med Chile 2005; 133 (12): 1500-3.

5. Armas Cruz R. Hernán Alessandri Rodríguez. Boletín Hosp Juan de Dios 1980; 27 (3), Editorial reproducida en: Neghme A. Hernán Alessandri Rodríguez. Médico, Maestro, Académico y Universitario. Su vida y su obra. Editorial Universitaria-Santiago de Chile: 1982. (Pags. 153-157). 